\title{
Anatomical and Functional Outcome of Rectangular Three Snip Punctoplasty in Primary Acquired Punctal Stenosis in a Tertiary Care Hospital of Karachi
}

\author{
Nazia Qidwai ${ }^{1}$, Muhammad Ashraf ${ }^{2}$, Mujahid Inam ${ }^{3}$, Adil Salim Jafri ${ }^{4}$, Saima Majid ${ }^{5}$ \\ ${ }_{1-5}$ Department of Ophthalmology, Isra Postgraduate Institute of Ophthalmology, Karachi
}

\begin{abstract}
Purpose: To find out the anatomical and functional outcomes of rectangular three-snip punctoplasty in primary acquired punctal stenosis.

Study Design: Quasi Experimental study.

Place and Duration of Study: Isra Postgraduate Institute of Ophthalmology, Karachi, from July to December 2020.

Methods: Patients of age 50 years and above, from either gender with primary acquired punctal stenosis of any grade (0-5) presenting with epiphora were recruited from Oculoplasty Outpatient department. Punctal stenosis with secondary causes like lesion on or around punctum or lacrimal drainage system and history of surgery or radiotherapy, lower canalicular or common canalicular stenosis were excluded from the study. Punctal stenosis was graded by Kashkouli scale and epiphora by the Munk score. After local anesthesia, two vertical cuts were given in the posterior wall of the punctum and a third cut was given horizontally to connect the vertical cuts. This resulted in a posterior ampullectomy. Syringing and probing was done to assess patency of the lower lacrimal drainage system. The patients were followed up for 6 months to assess for anatomical and functional success.
\end{abstract}

Results: At the end of six month anatomical success was achieved in $85 \%$ cases and functional success in $70 \%$ patients. Fifteen percent patients suffered re-stenosis of punctum.

Conclusion: Rectangular punctoplasty is a functionally successful procedure for treating primary acquired punctal stenosis. The anatomy is least distorted and long-term recurrence rate is also low.

Key Words: Epiphora, Primary acquired punctual stenosis, Punctoplasty, Munk score.

How to Cite this Article: Qidwai N, Ashraf M, Inam M, Jafri AS, Majid S. Anatomical and Functional Outcome of Rectangular Three Snip Punctoplasty in Primary Acquired Punctal Stenosis in a Tertiary Care Hospital of Karachi. Pak J Ophthalmol. 2021, 37 (2): 188-191.

Doi: http://doi.org/10.36351/pjo.v37i2.1166

\section{INTRODUCTION}

Punctal stenosis results in epiphora in $94 \%$ eyes. ${ }^{1}$ Incidence of acquired punctal stenosis has been

Correspondence: Nazia Qidwai

Department of Ophthalmology, Isra Postgraduate Institute of Ophthalmology, Karachi

Email:Nazia_qidwai@hotmail.com

Received: November 16, 2020

Accepted: January 27, 2021 reported to be between 8 and $54.3 \% .^{2}$ It can be idiopathic or secondary to chronic inflammation, eyelid malpositioning, infection (herpes zoster, chlamydia, human papilloma virus, and actinomyces), cicatricial conjunctivitis, trauma, eyelid neoplasms, use of topical antiglaucoma agents or chemotherapeutic agents such as Mitomycin C, aggressive lacrimal probing, tumors, porphyria cutanea tarda, acrodermatitis enteropathica, systemic chemotherapy, and irradiation and last but not the 
least, aging., ${ }^{3,4}$ These factors result in scarring and fibrosis. ${ }^{5,6}$ Punctal stenosis is graded according to the size of lumen of the punctum visible on slit lamp examination. Kashkouli et al suggested a scale of punctal stenosis. ${ }^{7}$ Various methods have been tried over the years to treat punctal stenosis. One snip procedure was first described by Bowman in $1853 .{ }^{8}$ As this did not relieve the symptom effectively, two, three and four snip punctoplasty were subsequently introduced. Three snip which is the most successful technique has two types, the triangular and the rectangular types. ${ }^{9}$ In the triangular type, a triangular flap is made by cutting the vertical canaliculus and then the horizontal canaliculus. The base of the canaliculus is then cut. In the rectangular type, however, two snips are made in the vertical canaliculus and a snip at the base. A posterior ampullectomy is hence created. ${ }^{9}$ Kim et al suggested yet another modification, the four snip punctoplasty. In this technique one snip is given in the vertical canaliculus followed by a horizontal snip. A third vertical or horizontal cut is given after which the base of the flap is removed. ${ }^{10,11}$ Various surgical techniques and adjunct therapies have thereby, been undertaken in a hope to improve surgical outcome. Adjunct therapies like use of mitomycin C, Kelly's and Reiss punctal punch, stents, punctal plugs, pigtail probe and placement of interrupted sutures after punctoplasty have been employed to aid management of complicated cases of punctal stenosis. ${ }^{12,13,14}$ Anatomical outcome of punctoplasty in various studies has been recorded to be $74.7 \%, 86 \%$ and $91 \% .^{15,16}$ Whereas, functional success was achieved in $64 \%$ in the study by Shahid et al and $89.8 \%$ in a study by Chak et al. ${ }^{9}$

Rationale of this study was to find out the functional and anatomical results in a tertiary care center of Karachi.

\section{METHODS}

Sample size was calculated through Rao Soft sample size calculator having a prevalence of $54.3 \%{ }^{17}$ of punctal stenosis with a $95 \% \mathrm{CI}$ and $5 \%$ margin of error. Non-probability convenient sampling technique was used. Punctal stenosis was defined as a condition in which the punctum was narrowed or occluded. Patients of 50 years and above age, with lower punctal stenosis of any grade (0-5) according to Kashkouli et al's scale of punctal stenosis, patent upper punctum, canaliculi and nasolacrimal duct as proven on syringing and probing through upper punctum and normal lower eyelid margin position were included in the study. Patients with past history of eyelids surgery, punctal stenosis with secondary causes like lesion on or around punctum or lacrimal drainage system, history of radiotherapy and lower canalicular or common canalicular stenosis were excluded.

This study was conducted in accordance with the ethical standards of the institutional and/or national research committee and with the 1964 Helsinki declaration and its later amendments or comparable ethical standards. Informed consent was obtained from all patients before surgery.

Before surgery, grading of punctal stenosis was done according to the Kashkouli et al's scale. ${ }^{3}$ Epiphora was graded according to the Munk score. ${ }^{18,19}$ Upper punctum was assessed for patency and then syringing and probing was performed via the upper punctum to assess the lacrimal drainage system. Local anesthesia was injected below the punctum and the standard rectangular three-snip procedure was performed. Two vertical cuts were given in the posterior wall of the punctum and a third cut was given horizontally to connect the vertical cuts. This resulted in a posterior ampullectomy. Syringing and probing was done to assess patency of the lower lacrimal drainage system. For punctal stenosis grade 0 , where no punctum could be found, a pigtail probe was used to identify the punctal site. Incision was given by the tip of a 1cc needle vertically at this identified site. Standard procedure for punctoplasty and syringing and probing was performed. Antibiotic eye drops were instilled and the patient was reviewed at $1^{\text {st }}$ week and then $1^{\text {st }}, 3^{\text {rd }}$ and $6^{\text {th }}$ months. On these follow up visits, improvement in subjective epiphora was assessed by Munk score and objectively by Kashkouli scale.

\section{The Munk score is as under.}

$0 \quad$ No epiphora

1 Occasional epiphora requiring drying or dabbing less than twice a day

2 Epiphora requiring dabbing two to four times per day

3 Epiphora requiring dabbing five to ten times per day

4 Epiphora requiring dabbing more than ten times daily or constant tearing

Kashkouli scale.

\begin{tabular}{cl}
\hline Grade & Clinical Findings \\
\hline 0 & No punctum (agenesis) \\
1 & Papilla is covered with a membrane (difficult to \\
2 & recognize) \\
& Less than normal size, but recognizable
\end{tabular}


Normal

Small slit $(<2 \mathrm{~mm})$

Large slit $(\leq 2 \mathrm{~mm})$

\section{RESULTS}

Two hundred and seventy two patients underwent rectangular punctoplasty. At the time of admission, $90 \%$ presented with epiphora and $10 \%$ with ocular discomfort. According to Kashkouli scale, punctal stenosis was of grade 2 in 210 patients, 42 had grade 1 and 20 had grade 0 punctal stenosis. Epiphora was graded by Munk score according to which 190 patients fell in grade 4, 30 fell in grade 3, 25 in grade 2 and 27 in grade 1 . Scoring was repeated after the procedure. At the end of $6^{\text {th }}$ month anatomical success was achieved in $85 \%$ cases and functional success in $70 \%$ patients. Presence of Epiphora despite punctual patency was in 10\%patients and $15 \%$ suffered restenosis of punctum.

\section{DISCUSSION}

Punctoplasty has developed over the years in the form of one to four snip procedures. Various studies have been conducted to assess results of the various types of punctoplasty. It is generally agreed that rectangular three snip punctoplasty has better results than the rest of the procedures in terms of anatomical and functional outcome. The purpose is to make the punctum patent again. By giving cuts in the vertical canaliculus and avoiding the horizontal canaliculus, we save the proximal lacrimal pump action.

The Triangular Three Snip procedure affects the lacrimal pump action. Chak et $\mathrm{al}^{9}$ showed more failures in the triangular punctoplasty group as compared to the rectangular one. Functional epiphora was commoner in the triangular punctoplasty group being $16.9 \%$ whereas in the rectangular punctoplasty group it was $10.2 \%$. Functional success in this study was $89.8 \%$. They concluded that rectangular 3-snip punctoplasty was less destructive procedure hence preserving the proximal lacrimal pump mechanisms. ${ }^{9}$

In triangular punctoplasty, the horizontal and vertical canaliculi are cut. This causes more anatomical destruction and prevention of the opposition and occlusion of the puncta and creation of an active pump and vacuum. In rectangular punctoplasty, however, only the vertical section of the canaliculus is excised. This preserves the anatomy and physiology of the lacrimal system. ${ }^{20}$
MJ Ali et al showed that $74.7 \%$ achieved functional success after rectangular punctoplasty. ${ }^{1}$ Caesar and McNab retrospectively evaluated 53 cases of punctal stenosis and found that $92 \%$ of patients showed subjective improvement in epiphora. ${ }^{4}$ Murdock et al found in their study that $86 \%$ were primarily asymptomatic following rectangular punctoplasty at an average of 1.2 months after surgery without the need for further surgical intervention. However, 14\% suffered restenosis and were dealt with secondary procedures using adjuncts which eventually elevated the success rate to $100 \% .^{17}$

Shahid et al showed $91 \%$ anatomical success and $64 \%$ functional success. However, $40.8 \%$ reported epiphora despite patent punctum. ${ }^{18}$ An audit conducted by Baig et al found $66.7 \%$ to improve functionally after rectangular punctoplasty. ${ }^{23}$

In our study $85 \%$ anatomical and $70 \%$ functional success indicates that rectangular punctoplasty is a good procedure for primary punctual stenosis. Restenosis in a small number of patients however, remains to be a concern and requires secondary surgical procedures including use of adjuncts. The various causes that have been attributed to punctual restenosis include chronic blepharitis, lid laxity and post-op cicatrisation. ${ }^{17}$

Limitation of this study is the single center trial and there was no control group. Further multi-center studies comparing different procedure should be carried out with long term follow-ups.

\section{CONCLUSION}

Rectangular three snip is a good procedure for primary acquired punctal stenosis. It provides long term relief from ephiora without causing any substantial complications.

\section{Ethical Approval}

The study was approved by the Institutional review board/ Ethical review board. (REC/IPIO/2020/003)

\section{Conflict of Interest}

Authors declared no conflict of interest.

\section{REFERENCES}

1. Ali MJ, Ayyar A, Naik MN. Outcomes of rectangular 3 -snip punctoplsaty in acquired punctal stenosis: is there a need to be minimally invasive? Eye, 2015; 29 (4): 515-518. 
2. Soiberman U, Kalkizaki H, Selva D, Leibovitch I. Clin punctal stenosis: definition, diagnosis, and treatment. Clin Ophthalmol. 2012; 6: 1011-1018.

3. Kashkouli MB, Beigi B, Murthy R, Astbury N. Acquired external punctal stenosis: etiology and associated findings. Am J Ophthalmol. 2003; 136 (6): 1079-1084.

4. Caesar RH, McNab AA. A brief history of punctoplasty: the 3-snip revisited. Eye Reconstr Surg. 2005; 19 (1): 16-18.

5. Port AD, Chen YT, Lelli GJ. Histopathological changes in punctal stenosis. Ophthal Plast Reconstr Surg. 2013; 29: 201-204.

6. Ali MJ, Mishra DK, Baig F, Lakshman M, Naik MN. Punctal stenosis: histology, immunology and electron microscopic features-a step towards unravelling the mysterious etiopathogenesis. Ophthal Plast Reconstr Surg. 2015; 31 (2): 98-102.

7. Kashkouli MB, Nilforushan N, Nojomi N, Rezaee R. External lacrimal punctum grading: reliability and interobserver variation. Eur J Ophthalmol. 2008; 18 (4): 507-511.

8. Bowman W. Method of treatment for epiphora depends on the overturning or obliteration of the tear points. Ann Oculist. 1853; 29: 52-55.

9. Chak M, Irvine F. Rectangular 3-snip punctoplasty outcomes: preservation of the lacrimal pump in punctoplasty surgery. Ophthal Plast Reconstr Surg. 2009; 25 (2): 134-135.

10. Kim SE, Lee SJ, Lee SY, Yoon JS. Outcomes of 4snip punctoplasty for severe punctal stenosis: measurement of tear meniscus height by optical coherence tomography. Am J Ophthalmol. 2012; 153 (4): 769-773.

11. Park SJ, Noh JH, Park KB, Jang SY, Lee JW. A novel surgical technique for punctal stenosis: placement of three interrupted sutures after rectangular three-snip punctoplasty. BMC Ophthalmology, 2018; 18: 70. https://doi.org/10.1186/s12886-018-0733-2.

12. MA'luf RN, Hamush NG, Awwad ST, Noureddin BN. Mitomycin C as adjunct therapy in correcting punctal stenosis. Ophthal Plast Reconstr Surg. 2002; 18 (4): $285-288$.

13. Wong ES, Li EY, Yeun HK. Long-term outcomes of punch punctoplasty with Kelly punch and review of literature. Eye (London, England). 2017; 31 (4): 560565.

14. Mandour SS, Said-Ahmed KE, Khairy HA, Elsawy MF, Zaky MA. A simple surgical approach for the management of acquired severe lower punctal stenosis. J Ophthalmol. 2019: 35618. https://doi.org/10.1155/2019/3561857
15. Murdock J, Lee WW, Zatezalo CC. Three-snip punctoplasty outcome rates and follow-up treatments. Orbit, 2015; 34 (3): 160-163.

16. Shahid H, Sandhu A, Keenan T, Pearson A. Factors affecting outcome of punctoplasty surgery: a review of 205 cases. Br J Ophthalmol. 2008; 92 (12): 1689-1692.

17. Bukhari A. Prevalence of Punctal Stenosis Among Ophthalmology Patients. Middle East Afr J Ophthalmol. 2009; 16 (2): 85-87.

18. Munk PL1, Lin DT, Morris DC. Epiphora: treatment by means of dacryocystoplasty with balloon dilation of the nasolacrimal drainage apparatus. Radiology, 177 (3): 687-690.

19. Ozlen RO, Levent A, Nesrin T, Onur K. Management of acquired punctal stenosis with perforated punctal plugs. Saudi J Ophthalmol. 2015; 29 (3): 205-209.

20. Lemp MA, Wolfley DE. The lacrimal apparatus. In: Hart WM, ed. Adler's Physiology of the Eye. 9th ed. Chapter 2. St. Louis, MO: Mosby-Year Book Inc, 1992.

21. Baig R, Aslam B, Ahmed K. An audit of 3-snip procedures performed at the Aga Khan University Hospital, Karachi, Pakistan. J Pak Med Assoc. 2020; 70 (3): 494-496.

\section{Authors' Designation and Contribution}

Nazia Qidwai; Assistant Professor: Concepts, Design, Literature search, Data acquisition, Data analysis, Statistical analysis, Manuscript preparation, Manuscript editing, Manuscript review.

Muhammad Ashraf; Associate Professor: Concepts, Design, Literature search, Data acquisition, Data analysis, Statistical analysis, Manuscript preparation.

Mujahid Inam; Assistant Professor: Concepts, Design, Literature search, Data acquisition, Manuscript preparation.

Adil Salim Jafri; Assistant Professor: Design, Literature search, Data acquisition, Manuscript editing, Manuscript review.

Saima Majid; Assistant Professor: Design, Literature search, Data acquisition, Manuscript editing, Manuscript review. 